\title{
Universal and non-universal aspects of wet granular matter under vertical vibrations
}

\author{
K. Huang ${ }^{1,2, a}$, K. Roeller ${ }^{1, b}$, and S. Herminghaus ${ }^{1, c}$ \\ 1 Max-Planck-Institute for Dynamics and Self-Organization, Bunsenstr. 10, 37073 Göttingen, Germany \\ 2 Experimentalphysik V, Universität Bayreuth, 95440 Bayreuth, Germany
}

\begin{abstract}
The phase diagram of vertically vibrated wet granular matter is investigated by both experiments and molecular dynamics type simulations, with a focus on the coexistence regime of the fluid and gas phases. Phase diagrams measured at various parameters including the rupture energy of liquid bridges, are presented. While for elastic grains, the transition from the fluid to the fluid-gas coexistence phase is found to be determined only by the rupture energy of liquid bridges [Fingerle et al. New J. Phys. 10, 053020 (2008)], inelasticity is found to introduce non-universal features into the phase diagram, which are also affected by the grain size.
\end{abstract}

\section{Introduction}

Many of the peculiar features of granular matter can be traced down to the dissipative nature of its grain scale dynamics. This gives rise to a wide variety of complex collective phenomena, and identifies granular matter as an interesting candidate for the investigation of physics far from thermal equilibrium. It has been proved useful to describe the collective behaviour of a large number of grains with notions borrowed from the physics of equilibrium phase transitions. For instance, granular matter can behave like a solid, a liquid, or a gas $[1,2]$, depending on the balance between energy injection and dissipation. As a particularly well controlled way of injecting energy, vertical vibration has been widely employed and explored in detail [3-12]. While 'classical' research on granular systems tried to avoid any side effects due to cohesion, the dynamical behavior of wet granular matter has been attracting more and more interest from physicists more recently [13-15]. The energy dissipation mechanism in wet granular matter is dramatically different from that of dry granular matter. In wet granular matter, the formation of capillary bridges between adjacent particles [16,17] gives rise to a well defined energy scale in the system, which is absent in dry granulates [14].

Since the interest of physicists has only recently turned to wet granular matter, its dynamical behavior is still much less well understood than that of its dry counterpart [14,15]. In a previous study [18], we investigated phase transitions of vertically vibrated wet granular matter by both experiments and molecular dynamics type simulations. Aside from a solid-fluid transition which is well known from dry granular systems, a second transition was found which leads to the formation of a gas phase. While the solid-fluid transition was determined by the force acting on the sample as in the dry systems, the fluid-gas transition was found to be driven by the injected energy. In the present study, we explore the scaling for the various transition lines with system parameters and try to distinguish what properties can be seen as 'universal' (depending

\footnotetext{
a e-mail: kai.huang@uni-bayreuth.de

b e-mail: klaus.roeller@ds.mpg.de

c e-mail: stephan.herminghaus@ds.mpg.de
} 


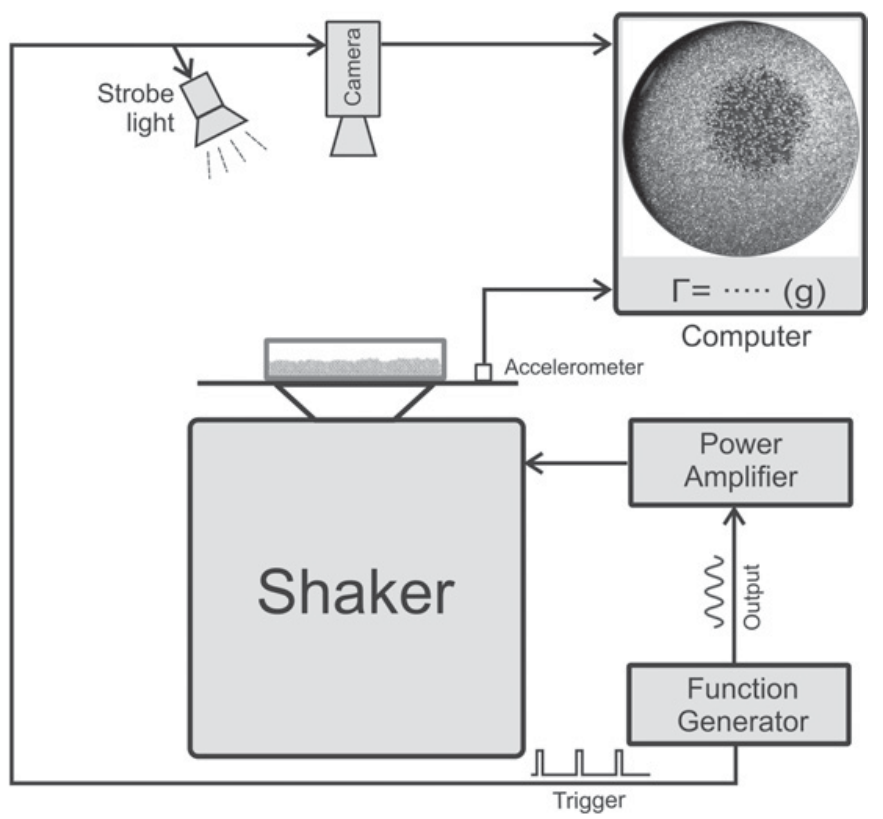

Fig. 1. Sketch of the experimental setup. Function generator, strobe light source and the high speed camera are synchronized with each other so that the images are captured at a certain phase of each vibration cycle. An example of a top view of the sample is shown in the inset photograph on the top right.

only on intrinsic parameters), and which have to be considered as non-universal (i.e., dependent on extrinsic parameters, like the volume of the container). In particular, we will address the question how the system properties are affected by inelastic effects in the impact between grains.

\section{Experimental setup}

Figure 1 shows a sketch of the experimental setup. Glass spheres with diameters $1.06 \mathrm{~mm}$, $1.22 \mathrm{~mm}$ and $1.5 \mathrm{~mm}$ (Whitehouse scientific, GP1060, GP1220, GP1500) are used as the granular sample. The width of the size distribution was about $10 \%$, which was sufficient to suppress crystallization. The glass beads were cleaned subsequently with ethanol, acetone, and Millipore water, and dried in an oven before use. The glass beads were placed into a flat cylindrical glass container, which was closed with a glass lid mounted on a separate stand. The latter fits well enough into the container to prevent glass beads from exiting, but enables free exchange of air, such that the gas pressure inside the cell was kept at atmospheric pressure. The height of the glass bead fill, $h$, was well below the total height of the cell, $H$, which could be adjusted by vertically moving the glass lid. A certain amount of wetting liquid was mixed into the sample before the experiment. Water, which has a surface tension of $\gamma \approx 72 \mathrm{mN} / \mathrm{m}$, was mostly used to wet the sample. Silicone oil (AK 5, Wacker; $\gamma \approx 21 \mathrm{mN} / \mathrm{m}$ ), was also used for comparison. Both liquids wet the glass surface well, with a contact angle below 10 degrees. The liquid content, $W$, is defined as the ratio between the volume of the added wetting liquid and the total volume the sample occupies (i.e., the area of the sample cell times the height of the granular pile, $h$ ). The container, which has a diameter of $145 \mathrm{~mm}$, was mounted on an electromagnetic shaker (Tira TV5880/LS). A sinusoidal signal from a function generator is used to drive the shaker. The strength of the vibration is measured by an accelerometer (Kistler 8702B100M1). The sample is illuminated with a strobe light (Helio Strobe Beta A2) and viewed from top by a high speed camera (PCO 1200s). The camera is externally triggered by the function generator so that the image is captured at the same phase of each vibration cycle. All measurements are taken after 
the wetting liquid is distributed homogeneously within the sample by shaking the sample at a low frequency $f<20 \mathrm{~Hz}$ and high acceleration $\Gamma \approx 10$ for a few minutes.

\section{Results and discussions}

\subsection{Observations}

As the frequency and amplitude of the drive are varied, a number of characteristic changes in the appearance of the sample are observed. At low acceleration, the glass beads just follow the harmonic vertical motion of the container, but no site exchange processes take place. This can be assessed most conveniently by the strobe light illumination, which reveals a completely static sample in this case. As the acceleration is increased, however, relative movements of the beads are observed, or even the appearance of a 'bubble' of a particularly 'hot' granular gas, surrounded by a 'fluid' (i.e., dense but mobile) phase [18]. We will explore in the present article the phase diagram of this system, both experimentally and by simulation, and try to distinguish its universal from non-universal features.

The phase diagrams are measured by keeping the driving frequency $f$ at a certain value and varying the peak vibration acceleration $\Gamma=4 \pi^{2} f^{2} A / g$, where $g$ is the gravitational acceleration and $A$ is the vibration amplitude. The transition from the solid ( $\mathrm{S}$ ) to the fluid $(\mathrm{F})$ phase is determined experimentally by first completely fluidizing the sample, followed by slowly decreasing $\Gamma$ until the particles stop moving. This criterion turned out to be better defined than just increasing $\Gamma$ and detecting the onset of particle motion: We assume this to be due to the difficulty to initialize the system with reproducible packing geometry. The transition from the fluid phase to the to the fluid-gas coexistence $(\mathrm{C}$ ) occurs at an acceleration where a 'gas bubble' nucleates (see the top view image embedded in Fig. 1). After nucleation, the (circular) granular 'gas bubble' grows spontaneously to a certain size. As $\Gamma$ is decreased again, the size of the gas bubble decreases continuously to zero, which indicates that there is a significant hysteresis for this transition. Within the hysteresis regime, the 'gas bubble' is unstable and fluctuates strongly in size. These fluctuations make a precise determination of the point of vanishing bubble size very difficult. We therefore rather define the F-C transition as the acceleration at which nucleation is observed. It is clear that the fluctuations of the gas bubbles close to the phase boundary are worthy of further investigations, which are currently under way. The transition from the fluid-gas coexistence $(\mathrm{C})$ to the gas $(\mathrm{G})$ phase again takes place at a well defined value of $\Gamma$, and leads to a completely homogeneous gaseous state of the sample. The abbreviations $\mathrm{S}$, F, C and G are henceforth used as abbreviations for solid, fluid, fluid-gas coexistence, and gas phases.

\subsection{Phase diagram: Experiments vs. simulations}

Figure 2 shows the experimentally determined phase diagram in both $\Gamma-f$ and $\Gamma-v$ planes, where $v=2 \pi A f$ is the maximum vibration velocity of the container bottom. The S-F boundary is located at $\Gamma \approx 1.9$, independent of the driving frequency $f$. It therefore lies higher than the corresponding transition for dry granular matter $(\Gamma \approx 1.2[2])$. This increase represents the enhanced mechanical stability of the granulate due to the network of capillary bridges forming between the grains. The independence of the critical acceleration upon the frequency of the driving underscores its physical significance. In fact, since the acceleration is directly related to a force, this transition has been identified as 'force driven' [18]. In the fluid-gas coexistence regime (C) (cf. photograph in Fig. 1), the size of the 'gas bubble' grows with the driving amplitude, while keeping its circular shape. During this growth, the surrounding fluid phase is pushed aside, and the height of the granular fluid increases until it reaches the top of the container. If the drive is increased further, the size of the 'gas bubble' saturates, and the density difference between gas and fluid phases decreases until the sample finally enters a homogeneous gas phase. This corresponds to reaching the boundary between the coexistence regime $(\mathrm{C})$ and the gaseous phase $(\mathrm{G})$. 

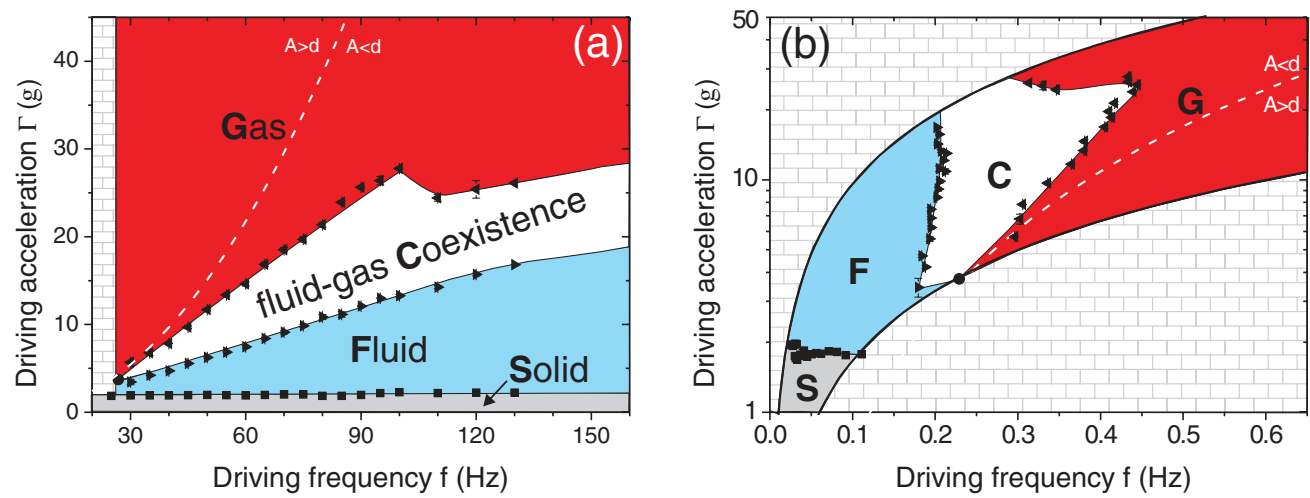

Fig. 2. Phase diagram of water-wet glass spheres with diameter $d=1.5 \mathrm{~mm}$, at a liquid content of $W=1 \%$ in $\Gamma f$ plane (a) and $\Gamma v$ plane (b). Filling height of the sample is $55 \%$ of the container height $H=6.6 \mathrm{~mm}$. Regions color coded by gray, blue, white and red correspond to solid (S), fluid (F), fluidgas coexistence $(\mathrm{C})$ and gas $(\mathrm{G})$ phases. The regions covered with brick patterns in the current and following plots are unexplored due to the limitation of the apparatus. The solid black dot corresponds to the converging point of the F-C and C-G transition lines. The dashed line indicates $A=d=1.5 \mathrm{~mm}$ ( $A$ is the vibration amplitude).

As shown in Fig. 2(b), the F-C transition occurs at a certain driving velocity (i.e., maximum velocity of the oscillating wall). This strongly suggests that this transition is linked to a certain characteristic velocity, or kinetic energy per particle. In fact, it has been successfully argued that the $(\mathrm{F})-(\mathrm{C})$ transition is determined by the balance between the energy required to break the capillary bridges between adjacent grains and the average kinetic energy of their center-ofmass motion [18]. As a simple mean-field model predicts, the (C)-(G) boundary should be just parallel to the $(\mathrm{F})-(\mathrm{C})$ boundary, at somewhat higher energy [18]. This is clearly not the case: the observed structure of the phase boundary is much more complex. Particularly prominent is the sharp backward bend which occurs at $\Gamma \approx 25 \mathrm{~g}=: \Gamma_{b}$. We will com back to this feature further below.

A glance back at Fig. 2(a) shows that the F-C and C-G transition lines in the phase diagram approach each other as the driving frequency decreases (Fig. 2(a)). They seem to intersect at $f_{c} \approx 27 \mathrm{~Hz}$ and $\Gamma_{c} \approx 3.6$. This point can be accurately detected by varying both the driving frequency and acceleration. We decrease $f$ continuously while keeping the size of the gas bubble maximal by varying $\Gamma$, until the gas bubble vanishes. The data points shown in Fig. 2 are averages of four measurements each, and the error bar is within the size of the data points. In an attempt to rationalize this finding, we just note that $f_{c}$ compares quite favorably with a natural time scale $t_{g}$ of the system, which can be derived from the attractive capillary force and the bead mass, $m$. Setting $t_{g}:=\sqrt{m / \gamma}$, we have $f_{g}=1 /\left(2 \pi t_{g}\right) \approx 45 \mathrm{~Hz}$, which is not far from $f_{c}$. This must clearly be investigated in further detail.

In a previous study [18], molecular dynamics type simulations based on two different models have been employed to study the phase diagram of elastically colliding particles. One of the models assumes that the attractive interaction between two particles which are connected by a liquid bridge is independent of the distance of the particles up to a certain distance at which the liquid bridge ruptures. This model has been termed the minimal capillary model. In contrast, the so-called thin-thread model has been used as well, which assumes the attractive force to be described by a delta-function located at the rupture distance. Both models consider the rupturing of liquid bridges as the only source of energy dissipation in the granular sample. In that case, the F-C transition should occur near $E^{*}=1$, where $E^{*}$ is the kinetic energy injected by one collision with the wall normalized by the rupture energy $E_{b} \propto R^{2} \gamma \sqrt{W}$ [18]. The most significant result was that the phase diagrams obtained with these two quite different force models were in almost perfect quantitative agreement. This can be seen as a justification to work with the thin-thread model, since this allows event-driven simulations which are much less time consuming than full integrations of an equation of motion with finite forces. In the present 

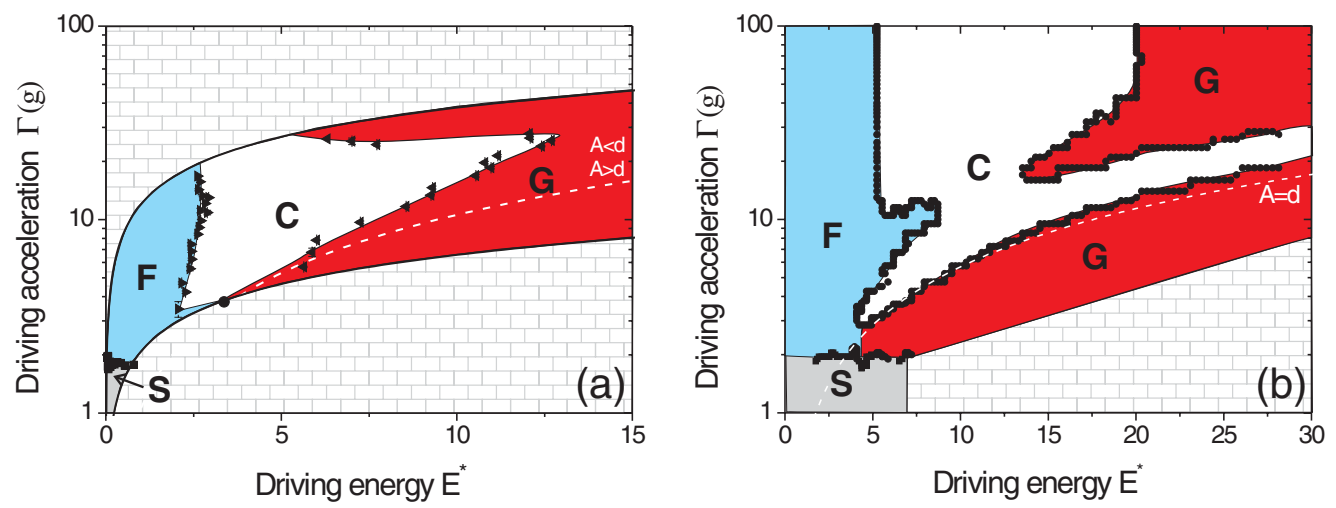

Fig. 3. Comparison of phase diagram between experiment (a) and simulation (b) in the $\Gamma-E^{*}$ plane, where $E^{*}$ is the energy injection rescaled by rupture energy of a liquid bridge. Restitution coefficient between particles $\epsilon_{p p}=0.8$, and between particle and container $\epsilon_{p w}=1$. The simulated system contains 1200 two-dimensional particles.

work, we therefore performed simulations using the thin thread model as in [18], but implement at the same time inelasticity in the particle collisions, in order to match the real experimental conditions more closely. We use a constant coefficient of restitution, $\epsilon$, which is defined as $\epsilon:=p_{f} / p_{i}$, where $p_{i}$ and $p_{f}$ are the momenta before and after the collision, respectively.

In the experimental phase diagram shown in Fig. 3(a), we see that the fluid-coexistence (F-C) transition occurs at $E^{*} \approx 2.5$, which is significantly larger than unity, and hence larger than obtained before in elastic simulations. We interpret this shift as being due to the influence of inelasticity in the collisions, frictional forces, and dimensionality [18] (simulation were in two dimensions). To support this view, we present in Fig. 3(b) simulations with inelastic impact dynamics $(\epsilon<1)$. Clearly, the F-C transition line is shifted to values of the normalized energy well above unity, which is in qualitative agreement with the experimental findings. Furthermore, the position of horizontal S-F transition line agrees quantitatively with experiment. An additional feature revealed by the simulation is that the fluid-gas coexistence region basically consists of two regimes. One elongated region appears at lower $\Gamma$ and approximately follows the line at which the vibration amplitude equals the particle diameter $A \approx d$. This seems to be a geometry-driven effect which is intimately connected to inelasticity, since in former simulation results with elastic particles [18] it was entirely absent. The other region, which is mainly bounded by two vertical lines corresponding to two well-defined driving energies, is located at higher $\Gamma$ and corresponds to the coexistence region as predicted by both earlier (elastic) simulations and mean-field theory [18]. In the intermediate $\Gamma$ region, both fluid-coexistence (F-C) and coexistence-gas (C-G) transitions are distorted, suggesting a superposition of both mechanisms.

\subsection{Scaling of the F-C transition}

Let us now investigate the variation of the positions of the transition lines as different system parameters are varied, in order to check the ideas put forward above. Fig. 4 shows phase diagrams for different liquid content $W$ and particle diameter. As the liquid content and particle diameter change, the rupture energy of liquid bridges, $E_{b} \propto R^{2} \gamma \sqrt{W}$, varies. We see that by choosing the rescaled driving energy, $E^{*}$, as the abscissa, the data for the F-C transition collapse on a single line within experimental scattering. This indicates that this transition is indeed dominated by the rupture energy alone.

We also investigated the influence of system dimensions on the observed phase transitions by varying the sample height $(h)$ and the container height $(H)$, as shown in Fig. 5. While the C-G transition line shifts substantially to the right as $H$ is increased while $h$ is kept constant, the F-C transition transition line remains almost unchanged. In contrast, changing the sample height, $h$, 


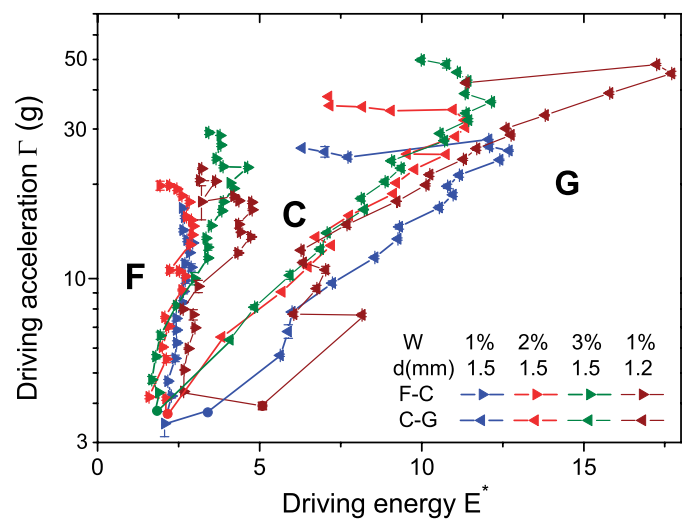

Fig. 4. Phase diagrams for water-wet glass spheres at different liquid content $W$ and particle diameters $d$ in the $\Gamma-E^{*}$ plane, where $E^{*}$ is the driving energy rescaled by the rupture energy of liquid bridges. Heights of the granular sample and the container are $h=3.6 \mathrm{~mm}$ and $H=6.6 \mathrm{~mm}$ correspondingly.

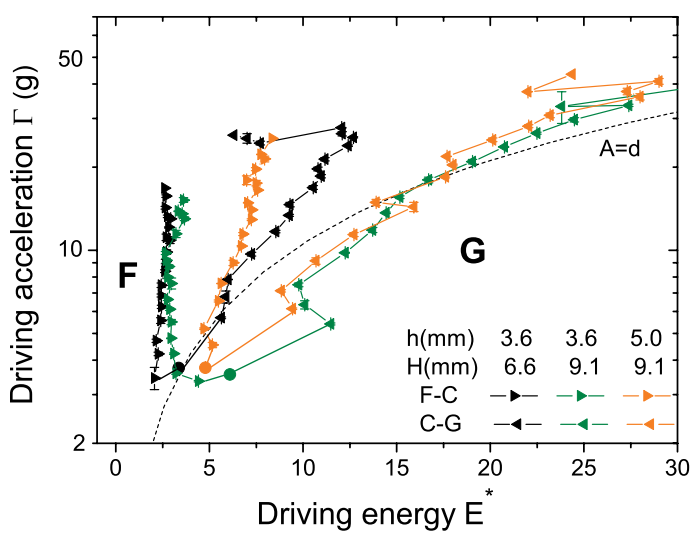

Fig. 5. Phase diagram for different sample height $h$ and container height $H$ for $1.5 \mathrm{~mm}$ glass spheres. The samples are wetted by water with liquid content $W=1 \%$.

at constant $H$ mainly affects the F-C transition, but not the C-G transition. We conclude that while the $\mathrm{C}-\mathrm{G}$ transition is determined by container size and should therefore be considered 'non-universal', the F-C transition is mainly governed by the sample itself. Furthermore, we may rationalize qualitatively how the dependence of the $\mathrm{F}-\mathrm{C}$ transition upon the height of the sample comes about. As is illustrated in Fig. 6, the shift of the F-C transition line may be due to the increased number of collisions involved in the injected energy's travelling from the container bottom (where it is injected) upwards through the sample. If the average number of collisions required for the energy to reach the top layer is $n$, the effective energy which reaches the top layer is $E_{t o p}=E_{\text {inj }} \epsilon^{2 n}$, where $\epsilon$ is the restitution coefficient of the collision between particles, and $E_{\text {inj }}$ is the energy injected through collisions between bottom layers of the sample and the container. If the energy of the top layer exceeds the capillary bridge energy, $E_{t o p}>E_{b}$, particles on top layers will be driven into a gaseous phase. For larger sample heights, $h$, the dissipation of energy within the sample increases and therefore more energy injection is needed to meet the criterion $E_{t o p}>E_{b}$. This may explain the shift of the fluid-coexistence (F-C) transition line to a higher value at increased $h$.

\subsection{Scaling of the C-G transition}

The behaviour of the $\mathrm{C}-\mathrm{G}$ transitions is more complex. This line consists of a lower branch which tends to follow the curve where the vibration amplitude equals the particle diameter, but there 


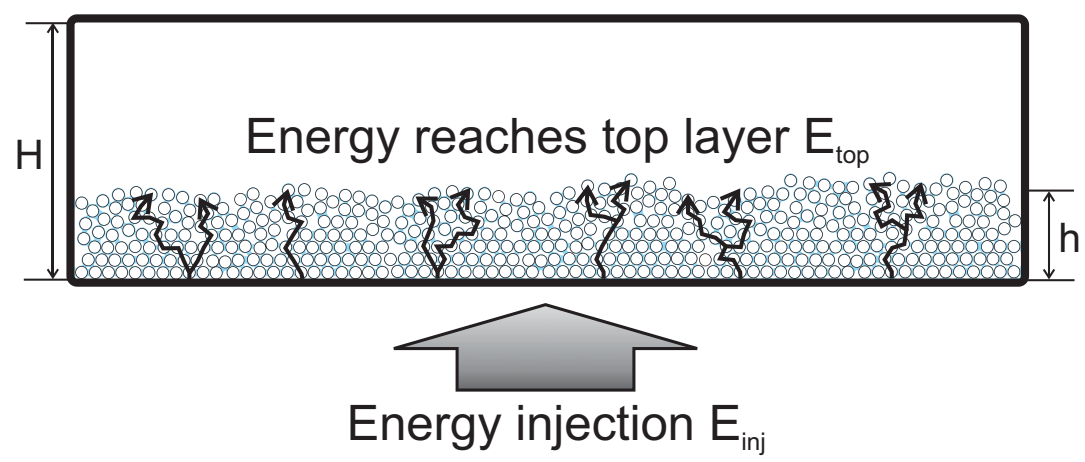

Fig. 6. A sketch showing how energy injection is transmitted upwards through collisions between neighboring particles. $h$ and $H$ are height of the sample and the container correspondingly.

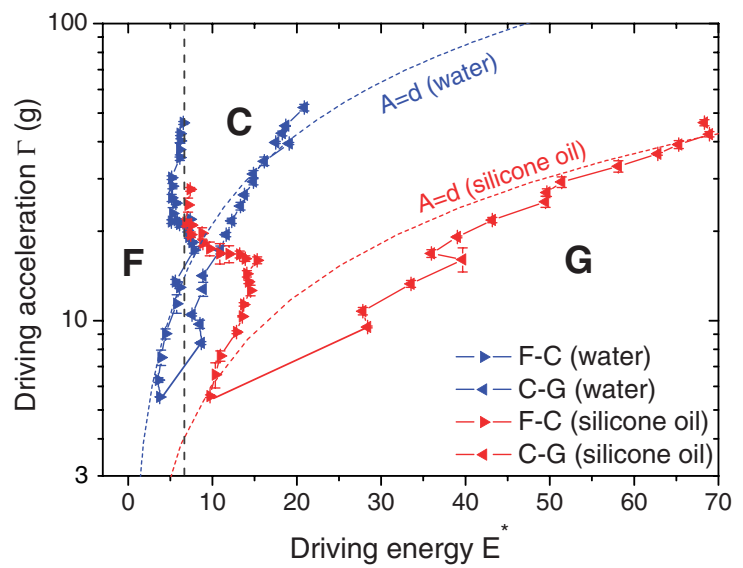

Fig. 7. Phase diagram of $1 \mathrm{~mm}$ glass spheres wetted by water and silicone oil in $\Gamma$ - $E^{*}$ plane. The surface tension of water is more than three times that of silicone oil. Other parameters are: liquid content $W=1 \%, h=3 \mathrm{~mm}$ and $H=9.1 \mathrm{~mm}$. Dashed lines correspond to $A=d$, the gray dashed line serves as a guide to the eye.

is as well a strong dependence on the height of the container, as already mentioned. This may be termed 'non-universal' behaviour, and we have already seen that inelasticity plays a vital role as well in shaping this transition line. Turning back to Fig. 4, we see that the horizontal upper branch shifts considerably to larger acceleration, $\Gamma_{b}$, as the liquid content is increased. This is in line with earlier simulations [18] which suggested that the sharp backward bend in the C-G transition line occurs when the vibration amplitude equals the rupture distance of the capillary bridges. The latter is increased as the liquid content increases, which would be consistent with an upshift of the horizontal upper boundary of the coexistence region. This aspect needs further investigation.

Finally, we have varied the surface tension of the wetting liquid to check the concepts put forward above. As shown in Fig. 7, phase diagrams for different wetting liquid are investigated for $d=1 \mathrm{~mm}$ particles. Again, we observe approximate data collapse for the F-C transition above the curve defined by $A=d$. This confirms that the scaling with rupture energy of liquid bridges in this region is appropriate. Again, the transition line between the $\mathrm{C}$ and $\mathrm{G}$ phases seems to be dominated by geometric effects, such as the equality of $A$ and $d$.

\section{Conclusions}

To summarize, we have confirmed by experimental studies as well as by simulations taking inelasticity into account that the phase diagram of mechanically agitated wet granular 
matter shows two universal phase transitions which depend only on few general intensive system parameters. One is the solid-fluid (S-F) transition which is driven by the applied force scale, represented here by the applied acceleration. The position of this transition line is directly given by the adhesive force acting between adjacent grains. The second is the transition between a fluid (F) phase and fluid-gas coexistence (C), and is driven by the injected kinetic energy. Its position is given by the energy which propagates from the bottom of the pile (where agitation occurs) to the upper surface of the sample, where evaporation and thereby the transition to coexistence is taking place. The relevant energy scale is the energy which is needed to break a liquid bridge between two adjacent grains. Aside from these two transitions, which in the plane spanned by the acceleration and agitation velocity are represented by a horizontal and a vertical straight line, respectively, there are as well a number of non-universal features, which mainly show up in the transition from fluid-gas coexistence to the gas phase. These depend notably on geometric relations like the size of the container, and are furthermore strongly dependent on the inelasticity of the grains.

\section{References}

1. H.M. Jaeger, S.R. Nagel, R.P. Behringer, Rev. Mod. Phys. 68, 1259 (1996)

2. J. Duran, Sands, Powders and Grains (An Introduction to the Physics of Granular Materials) 1st edn. (Springer-Verlag, New York, Inc., 2000)

3. F. Melo, Phys. Rev. Lett. 75, 3838 (1995)

4. W. Losert, D.G.W. Cooper, J.P. Gollub, Phys. Rev. E 59, 5855 (1999)

5. P.B. Umbanhowar, F. Melo, H.L. Swinney, Nature 382, 793 (1996)

6. A. Goetzendorfer, J. Kreft, C.A. Kruelle, I. Rehberg, D. Svensek, Phys. Rev. Lett. 95, 135704 (2005)

7. A. Goetzendorfer, C.A. Kruelle, I. Rehberg, D. Svensek, Phys. Rev. Lett. 97, 198001 (2005)

8. I.S. Aranson, L.S. Tsimring, Rev. Mod. Phys. 78, 641 (2006)

9. K. Huang, G.Q. Miao, P. Zhang, R.J. Wei, Phys. Rev. E 73, 041302 (2006)

10. P.M. Reis, R.A. Ingale, M.D. Shattuck, Phys. Rev. Lett. 96, 258001 (2006)

11. A. Prevost, P. Melby, D.A. Egolf, J.S. Urbach, Phys. Rev. E 70, 050301 (2004)

12. R.D. Wildman, D.J. Parker, Phys. Rev. Lett. 88, 064301 (2002)

13. L. Bocquet, E. Charlaix, F. Restagno, C. R. Physique 3, 207 (2002)

14. S. Herminghaus, S., Adv. Phys. 54, 221 (2005)

15. N. Mitarai, F. Nori, Adv. Phys. 55, 1 (2006)

16. M. Scheel, D. Geromichalos, S. Herminghaus, J. Phys. Condens. Matter 16, S4213 (2004)

17. M. Scheel, R. Seemann, M. Brinkmann, M. Di Michiel, A. Sheppard, B. Breidenbach, S. Herminghaus, Nat. Mat. 7, 189 (2008)

18. A. Fingerle, K. Roeller, K. Huang, S. Herminghaus, New J. Phys. 10, 053020 (2008) 